\title{
Development and Optimization of Antennas for HDTV Reception
}

\author{
A. Raizer \\ L. B. Lazare
}




\title{
Development and Optimization of Antennas for HDTV Reception
}

\author{
A. Raizer and L. B. Lazare
}

\begin{abstract}
This paper presents the development and optimization of antennas for reception of high-definition digital television (HDTV). An efficient simulation method will be presented, which uses finite element software to develop antennas without the need for complex calculations. The comparisons were made based on the main parameters related to the antennas, in order to establish a better choice both from the technical and economic point of view.
\end{abstract}

Index Terms-Development and Optimization of Antennas, HDTV, Numerical Simulation.

\section{INTRODUCTION}

$\mathrm{C}$ UnRENTLY, high definition digital television (HDTV) is an important television broadcasting system in Brazil due to the large investments for the transition from analogue to digital television. To carry out the transmission and reception of the HDTV signals, UHF band (Ultra High Frequency) is used, more specifically between the frequencies of $470 \mathrm{MHz}$ and $806 \mathrm{MHz}$. A crucial item in transmission and reception systems for digital TV signals is the receiving antenna. Therefore, HDTV antenna solutions with efficient reception and low production cost are sought.

An omnidirectional antenna is important for the HDTV reception system [1], because for non-satellite transmission systems, the transmitted signal is not directional and the receiving antenna must receive signals from several directions, ideally from all directions.

From the reception efficiency point of view, is required an antenna that couples the highest possible power as well can receive signals from several directions, whether installed indoors or outdoors. From the point of view of production cost, a small antenna that uses less material is required.

According these characteristics, it is presented in this paper the development and optimization of four antennas models for HDTV. A loop antenna and an antenna with meander Line structure based on [1] were developed for HDTV. The antennas improvement process and an antenna obtained from

This paper was supported financially by the company W3Sat through the research project titled "Development of antennas for reception of digital signals on high definition television (HDTV)".

A. Raizer (adroaldo.raizer@ufsc.br) and L. B. Lazare (lucas.lazare@grad.ufsc.br), are within GEMCO - Engineering and Electromagnetic Compatibility Group; MagLab - Electromagnetism and Electromagnetic Compatibility Laboratory; Federal University of Santa Catarina (UFSC); P.O.Box 5024; Florianópolis-SC, Brazil, ZIP Code 88040970 . the junction of the developed antennas are presented, as the simulation of electrical characteristics and parameters. A comparison between the performances of the four antennas is also presented.

In order to reach the desired results, a software based on the finite element method (FEM) [2][3] was used, significantly reducing the development time of the process.

\section{Magnetic Dipole Antenna}

The magnetic dipole antenna (loop) was developed based on the theory exposed in [4]. It is constituted by a radiant element that is divided, usually in the center, to allow a feeder to apply energy from a transmitter, or to be transferred to a receiver. The length of the radiant element determines many of the dipole antenna properties, such as impedance and central frequency of operation. This is an important characteristic of the antenna, which allows various configurations and antenna formats.

\section{A. Antenna Presentation}

The loop antenna is based on the theory of folded dipoles. As presented in [1], the length of the antenna, or loop, is directly proportional to the wavelength as well its area. As also presented in [1], one of the ways to increase the resistance of a loop antenna is by adding other loops to it. Therefore, knowing that the frequency range of Digital TV is from 470 $\mathrm{MHz}$ to $806 \mathrm{MHz}$, and using the concepts mentioned, the antenna developed is shown in Fig. 1. The antenna was constructed in stainless steel with thickness of $0.8 \mathrm{~mm}$.

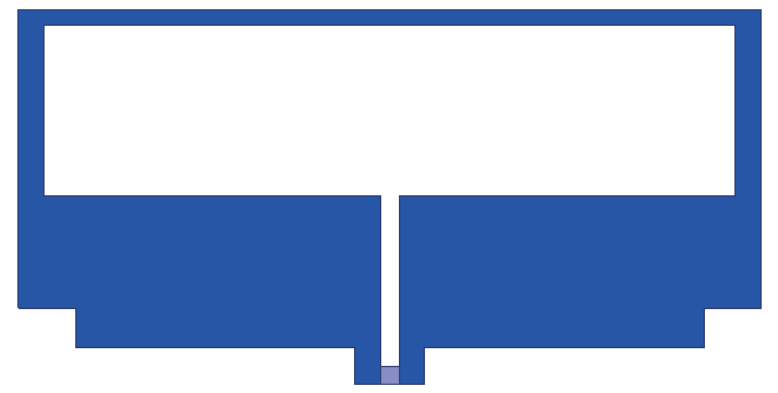

Fig. 1. Loop antenna.

\section{B. Modeling and Simulation}

To verify the performance of the antenna, its model was simulated using software based on the finite element method [3] in frequency range of HDTV. It was simulated the return loss, Voltage Standing Wave Ratio (VSWR), Smith chart, antenna impedance and radiation patterns. 


\section{1) Return Loss and VSWR}

The return loss, measured in decibel $(\mathrm{dB})$, of an antenna shows the relationship between the power reflected and the power transmitted. It is a relation that arises from the measure of $S_{11}$ parameter [5]. Thus, with a reference impedance of 75 $\Omega$, the loop antenna has the return loss shown in Fig. 2 .

A return loss of $\leq-10 \mathrm{~dB}$ is conventionally considered satisfactory, which guarantee $\geq 90 \%$ power transfer. Therefore, this will be the criterion used to determine if the antenna performs satisfactorily or not in HDTV frequency range.

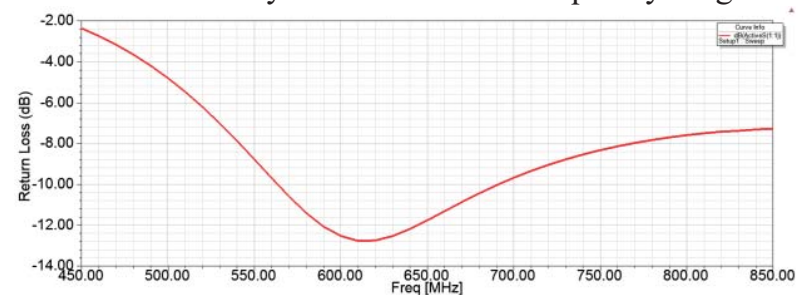

Fig.2. Loop antenna return loss.

As shown in Fig. 2, is observed that the antenna performs satisfactorily only in the range of $562 \mathrm{MHz}$ to $690 \mathrm{MHz}$. However, it is expected to obtain the return loss below $-10 \mathrm{~dB}$ for the entire Digital TV frequency range.

\section{2) Smith Chart and Antenna Impedance}

The Smith chart is a graphical system that visually displays the impedance of an antenna for a frequency range. It may present normalized impedance through a reference, which is the most common case, or it may present a non-normalized impedance [4]. Based on nominal impedance of TV system, the Smith chart of $75 \Omega$ will be presented on this paper.

Knowing that the circles represent constant resistances and the curves represent constant reactances, it is desirable that the antenna impedance curve be as close as possible to the unity resistance circle and the zero reactance curve. The normalized Smith chart to $75 \Omega$ of the original shaped loop antenna is shown in Fig. 3 for frequency range of $450 \mathrm{MHz}$ to $850 \mathrm{MHz}$.

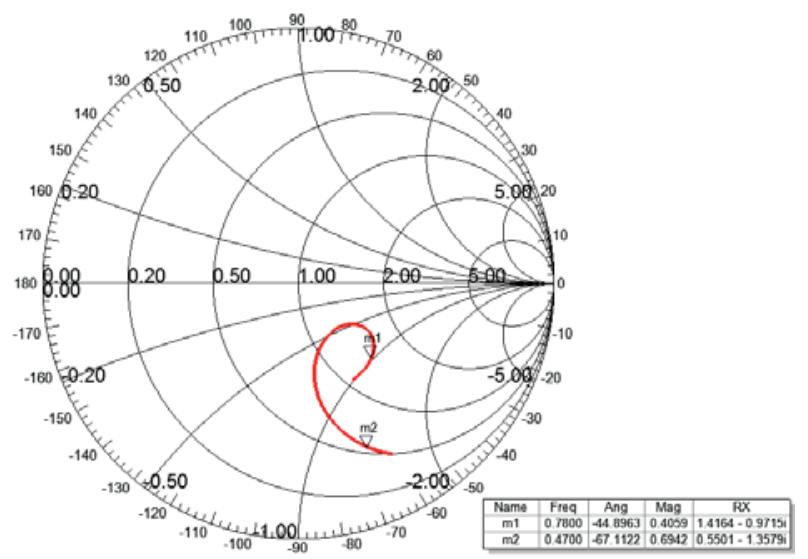

Fig.3. Loop antenna Smith chart.

It is noted that the antenna has a resistance close to $75 \Omega$ for almost the entire HDTV frequency range, but the antenna has a high negative reactance practically the same frequency range. The resistance and reactance of the antenna are shown in Fig. 4 and Fig. 5, respectively.

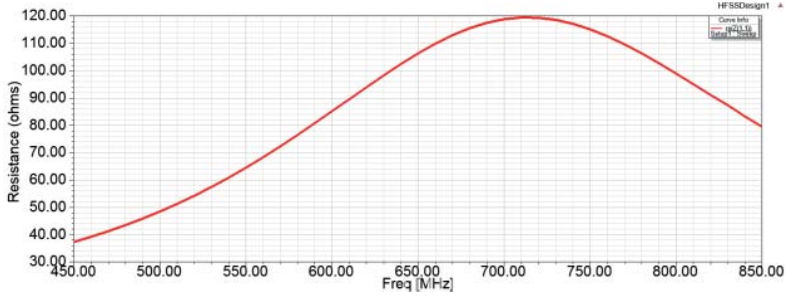

Fig.4. Loop antenna resistance.

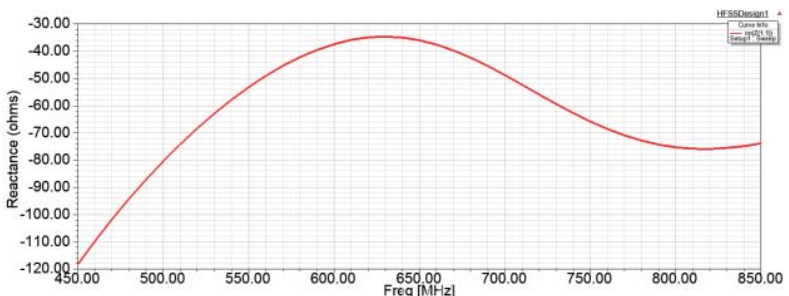

Fig. 5. Loop antenna reactance.

\section{3) Radiation Patterns}

The radiation patterns of an antenna graphically show the gain and the directivity of the antenna in measured directions [4]. These patterns usually present the gain value for each direction and are plotted in two dimensions. The spherical coordinate system is used as a reference for plotting and the same graph shows the curve for $\Phi=0^{\circ}$ and for $\Phi=0^{\circ}$, while the angle $\theta$ varies in $360^{\circ}$.

The radiation pattern of loop antenna for frequency of 470 $\mathrm{MHz}$ and $806 \mathrm{MHz}$ are shown in Fig. 6 and Fig. 7, respectively.

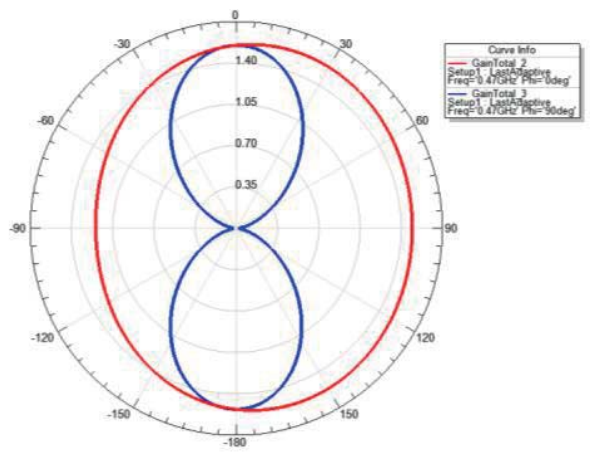

Fig. 6. Loop antenna radiation pattern for $470 \mathrm{MHz}$.

It is observed that the antenna has practically an omnidirectional behavior, which is desirable when the direction of the signal reception is not known.

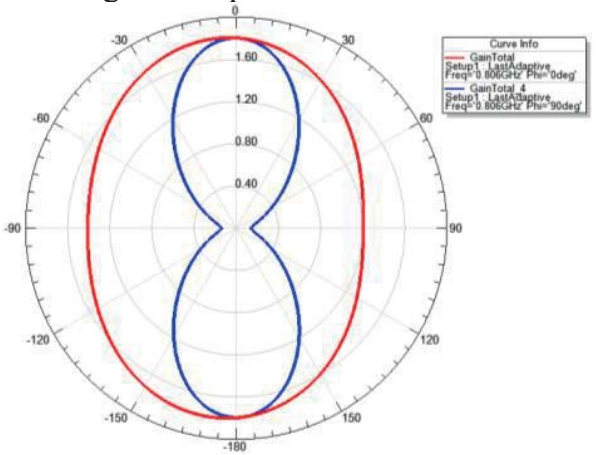

Fig. 7. Loop antenna radiation pattern for $806 \mathrm{MHz}$. 


\section{LOOP ANTENNA OPTIMIZATION}

The first step to improve the antenna is to analyze the shape of the return loss curve shown in Fig. 2 and check if there is any behavior that can be used. It can be observed that there is a resonance at approximately $615 \mathrm{MHz}$. At frequencies greater than that, the return loss increases slowly compared to the increase in the return loss at frequencies smaller than the resonance frequency. It is possible to decrease the resonance frequency of the antenna to better take advantage of this feature of the return loss curve of the original loop antenna.

In order to reduce the resonance frequency, was used the direct relation between the loop length and the area of the antenna with the wavelength for resonance. Therefore, in order to decrease the resonant frequency, that is, to increase the resonant wavelength, the length of the loop must increase.

With an increase of $60 \mathrm{~mm}$ in the length of the loop according to the model of the antenna shown in Fig. 8, return loss shown in Fig. 9 was obtained.

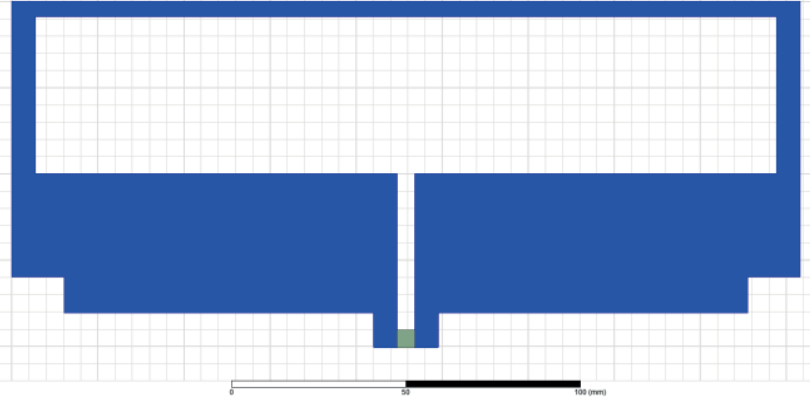

Fig. 8. Loop antenna with $60 \mathrm{~mm}$ increased length.

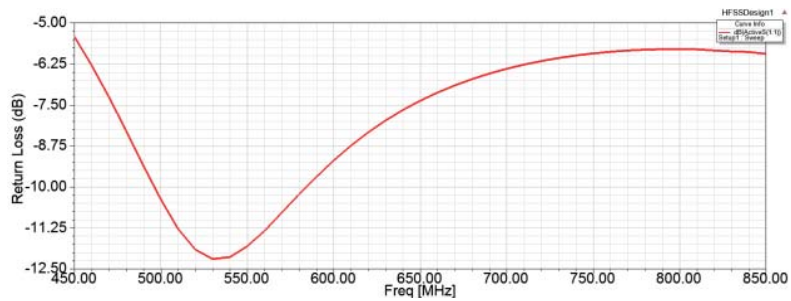

Fig. 9. Return loss with increased length.

It is observed that, as expected, the resonance frequency has decreased approximately to $530 \mathrm{MHz}$. It is also noted that for frequencies greater than the resonance frequency there is a slow decay, but there is still an undesirable return loss for the most frequency range of HDTV. This is explained by the Smith chart of this new antenna, as shown in Fig. 10.

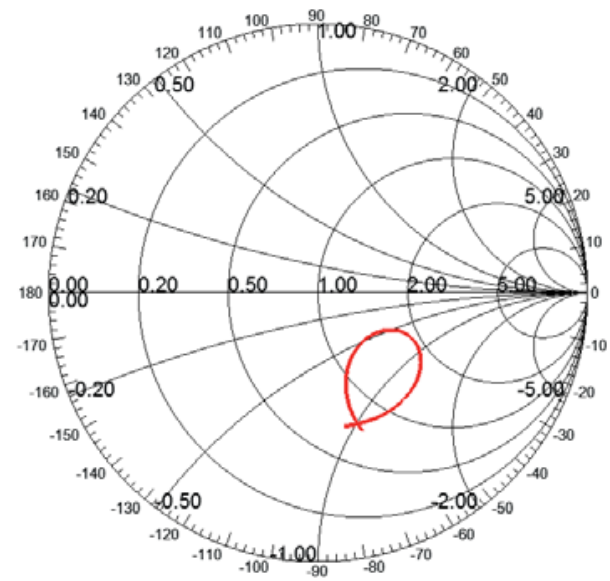

Fig. 10. Smith chart with increased length.

It is observed that the antenna continues with a negative reactance for most frequencies of operation. However, it can also be seen, compared to the Smith chart of the initial antenna shown in Fig. 3, the curve is with a smaller variation of the impedance in HDTV frequency range. This characteristic is important because the curve is centered on a point of the unit resistance circle of the Smith chart. Therefore, it is only necessary to complement this negative reactance with a positive one, that is, with a series inductor element, so that the reactance increases as much as possible to the largest frequency range possible.

Observing the Smith chart shown in Fig. 10, it can be concluded that the inductive element have an average normalized impedance at $75 \Omega$ of approximately $0.8 \mathrm{i} \Omega$.

A method to match the impedance by adding an inductive element is shown in [1]. It is a method based on stubs, which consists of increasing the length of loop antenna terminal. The loop antenna terminal would be the place where the antenna feed is done. Therefore, using this technique, tests have been done. Initially it was attempted to increase the length of the terminal by $20 \mathrm{~mm}$ according to the antenna shown in Fig. 11. With this, the return loss shown in Fig. 12 was obtained.

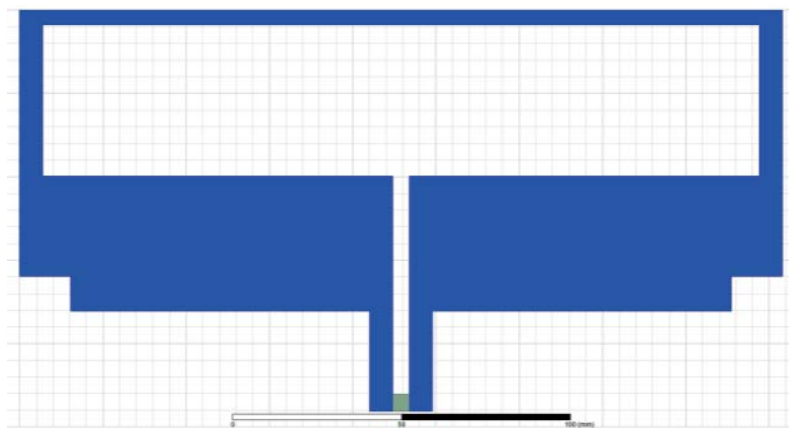

Fig. 11. Loop antenna with increase of $60 \mathrm{~mm}$ in length and increase of 20 $\mathrm{mm}$ in terminal. 


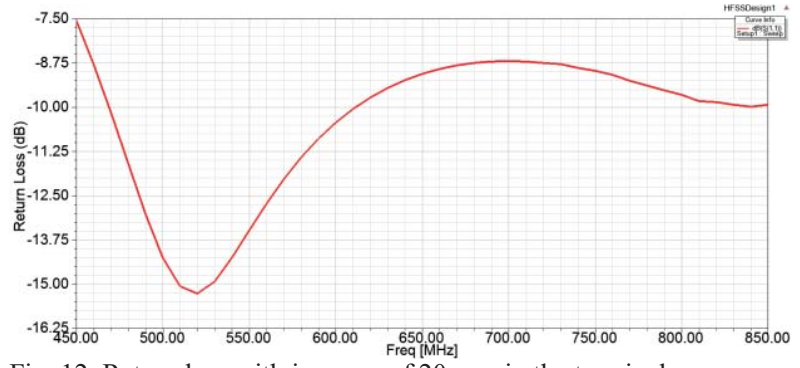

Fig. 12. Return loss with increase of $20 \mathrm{~mm}$ in the terminal.

It is noted that a generalized decrease of the return loss was achieved with a significant improvement in the antenna performance. However, there is still a frequency range that does not meet the return loss requirement of less than $-10 \mathrm{~dB}$. This is again explained by Smith chart of that antenna with a longer loop length and a longer terminal length as shown in Fig. 13.

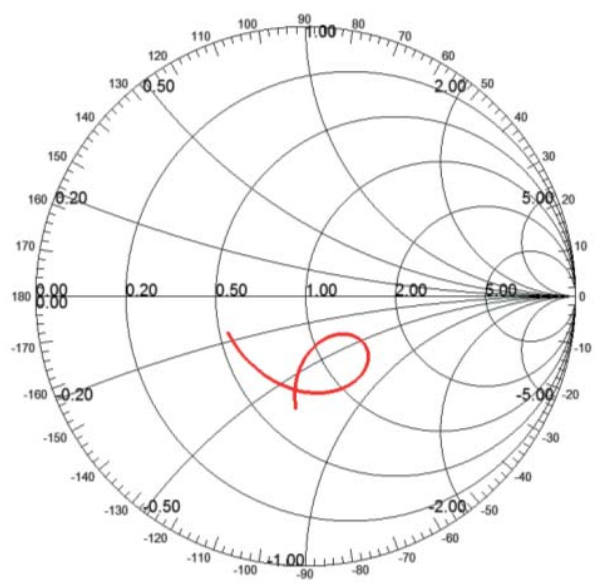

Fig. 13. Smith chart with $20 \mathrm{~mm}$ increased in the terminal.

Although there is an increase in the value of the reactance for the HDTV band, it is not yet centered at zero. Thus, the next step was to increase the terminal length even further. Therefore, an increase of more $20 \mathrm{~mm}$ corresponding to an increase of $40 \mathrm{~mm}$ in relation to the original loop antenna was made. Thus, the antenna model obtained is shown in Fig. 14 and the return loss for this new antenna is shown in Fig. 15.

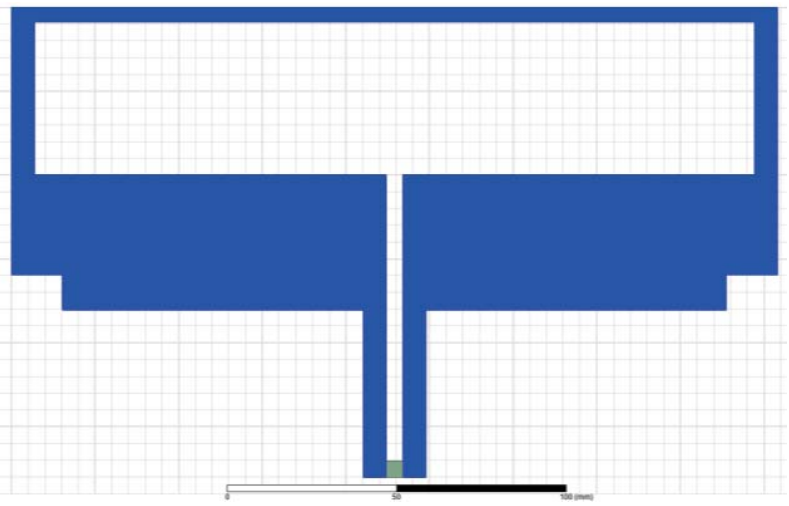

Fig. 14. Loop antenna with $60 \mathrm{~mm}$ increased in length and increased $40 \mathrm{~mm}$ in the terminal.

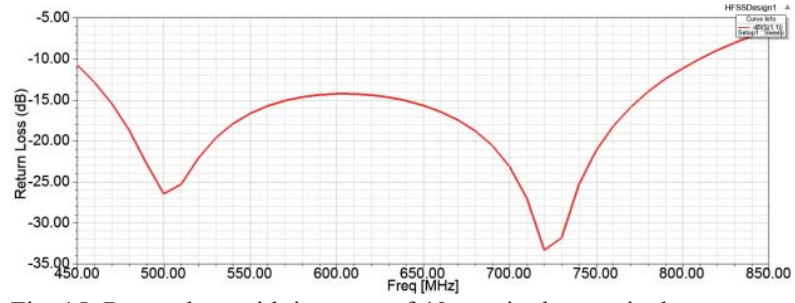

Fig. 15. Return loss with increase of $40 \mathrm{~mm}$ in the terminal.

As can be observed in Fig. 15, there was again a generalized improvement in the return loss of the antenna, with values below $-10 \mathrm{~dB}$ for the entire HDTV frequency range. This is explained by observing the Smith chart to this new antenna shown in Fig. 16.

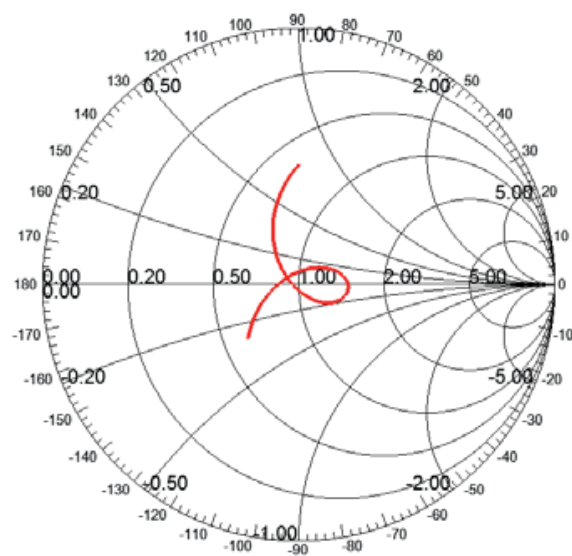

Fig. 16. Smith chart with increase of $40 \mathrm{~mm}$ in the terminal.

It is observed that the initial objective is reached, getting a close resistance 1 and a close reactance 0 for most of the simulated frequency range getting a very good impedance matching. Also tried to further increase the length of the antenna terminal, however this causes deterioration in impedance matching.

It is important to note the change that there are in the radiation patterns in relation to the initial loop antenna. The radiation patterns of new loop antenna with $60 \mathrm{~mm}$ increased in length and $40 \mathrm{~mm}$ in the terminal compared, for the frequencies of $470 \mathrm{MHz}$ and $806 \mathrm{MHz}$ are shown in Fig. 18 and Fig. 17, respectively.

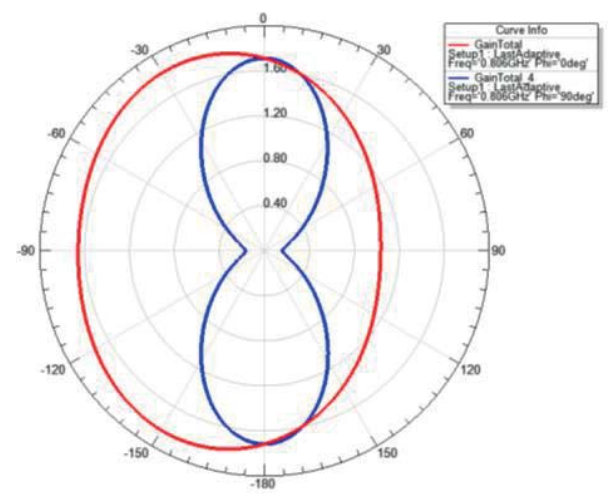

Fig. 17. Antenna radiation pattern for $806 \mathrm{MHz}$ with $40 \mathrm{~mm}$ increased in terminal. 


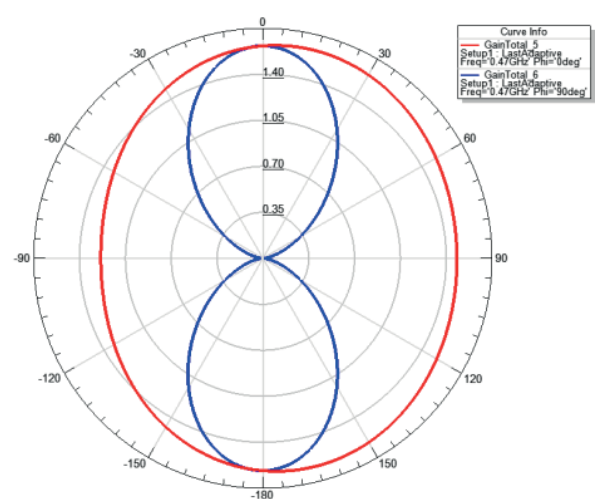

Fig. 18. Antenna radiation pattern for $470 \mathrm{MHz}$ with $40 \mathrm{~mm}$ increased in terminal.

It is observed that although the format of the radiation pattern has changed a bit due mainly to the change in the antenna terminal, the gains have remained practically the same.

\section{MEANDER Line Electric Dipole ANTENNA}

An electric dipole antenna was developed based on theory expose in [6], with inclusion of a PCB (Printed Circuit Board) for connection of the antenna connector with a coaxial cable.

\section{A. Antenna Presentation}

The developed antenna has the same characteristics as the presented in [6], which is based on the concept of electric dipole antennas, as well as meander line antennas. For connection of the antenna to HDTV reception system, it is necessary to use a radio frequency connector on a PCB. The used connector is shown in Fig. 19. It has four pins on the sides that connect to the reference or ground of the antenna and a center pin that connects to the antenna signal.

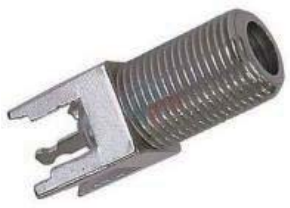

Fig. 19. Radio frequency connector.

A PCB was modeled through software [3] which has appropriate connections for both the antenna and the connector which is shown in Fig. 20.

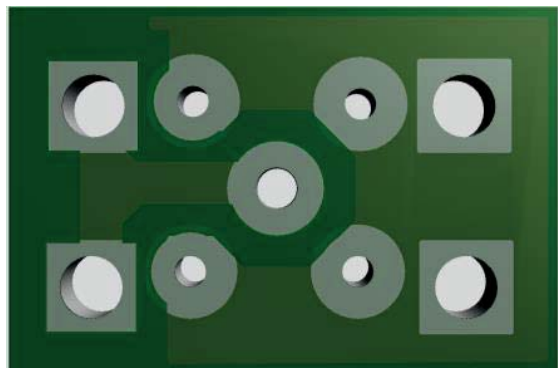

Fig. 20. PCB Project.

\section{B. Simulation Results Including the PCB}

Using the software [3], the meander line electric dipole with PCB inclusion was simulated, as shown in Fig. 21. With this, it was possible to obtain the most important antenna parameters such as radiation patterns, return loss and Smith chart, with reference impedance for matching as $75 \Omega$.

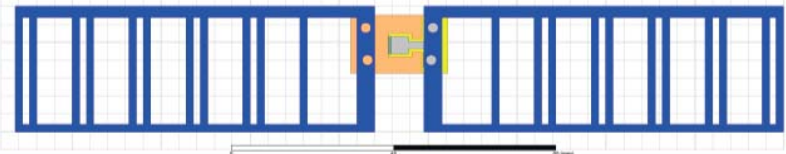

Fig. 21. Meander line electric dipole with PCB.

In Fig. 22 can be observed that the antenna return loss, with a similar behavior to the original loop antenna presented. This is explained by the impedance mismatch that exists for a certain frequency range presented by the Smith chart of Fig. 23.

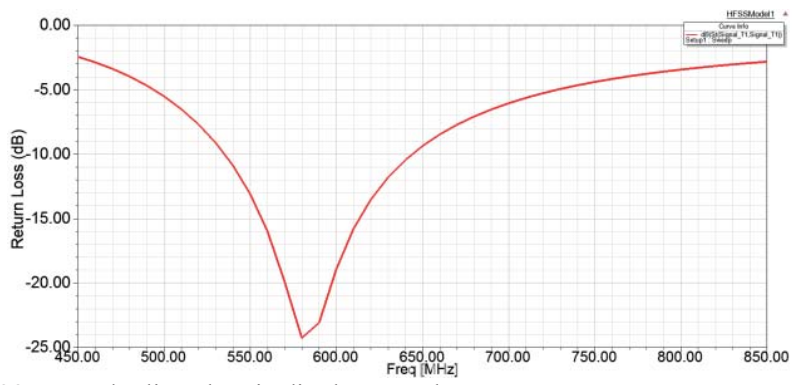

Fig. 22. Meander line electric dipole return loss.

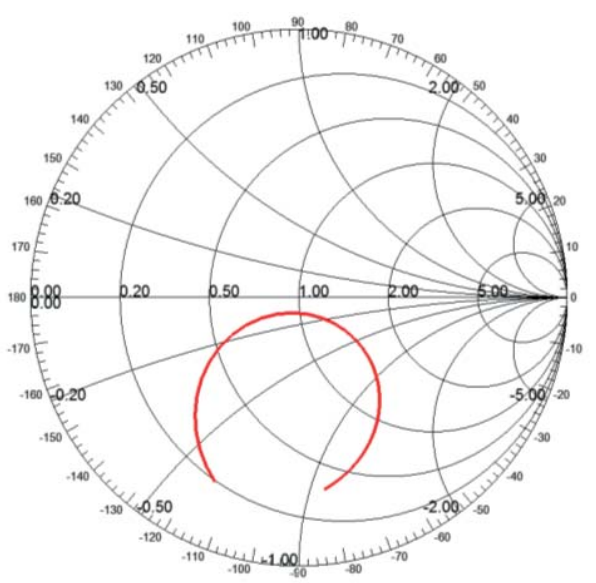

Fig. 23. Smith chart of the meander line electric dipole antenna for frequencies from $450 \mathrm{MHz}$ up to $850 \mathrm{MHz}$.

The radiation patterns for this antenna at the frequencies of $470 \mathrm{MHz}$ and $806 \mathrm{MHz}$ are shown in Fig. 24 and Fig. 25, respectively. 


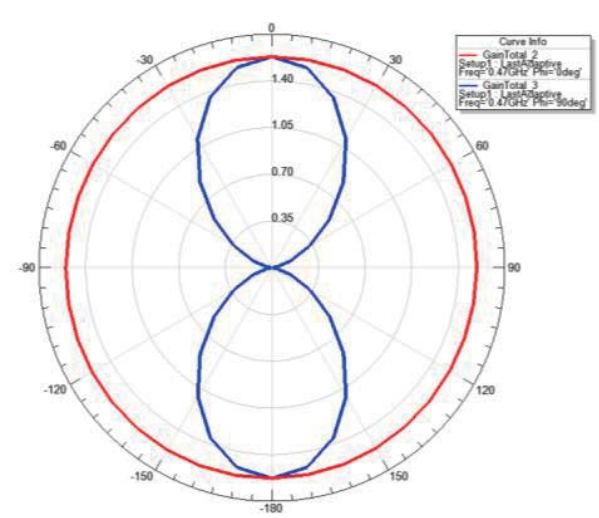

Fig. 24. Meander line electric dipole antenna radiation pattern for $470 \mathrm{MHz}$.

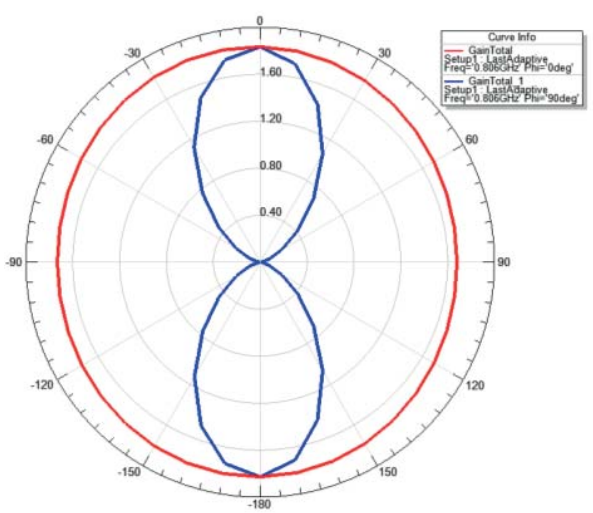

Fig. 25. Meander line electric dipole antenna radiation pattern for $806 \mathrm{MHz}$.

It is observed that the radiation pattern is practically equal to that of an electric dipole, that is, it is an omnidirectional antenna and has a gain greater than a standard electric dipole.

\section{Magnetic Loop Antenna With Meander Line}

After obtaining the results with the modified loop antenna in the return loss and verifying the omnidirectional behavior of the meander line electrical dipole antenna, the two antennas were united to take advantage of the individual characteristics in only one antenna. This union was made and modeled as shown in Fig. 26.

Using the software [3], simulation was made and obtained results of radiation patterns, return loss, Smith chart for this antenna. The return loss and Smith chart are presented in Fig. 27 and Fig. 28, respectively. Radiation patterns for the 470 $\mathrm{MHz}$ and $806 \mathrm{MHz}$ frequencies are shown in Fig. 29 and Fig. 30 , respectively.

It is observed that, just like the modified loop antenna, the presented antenna maintains a return loss below $-10 \mathrm{~dB}$ for the entire frequency range used for HDTV. This is explained by the impedance matching with the nominal impedance of the television systems, presented by the Smith chart in Fig. 28.

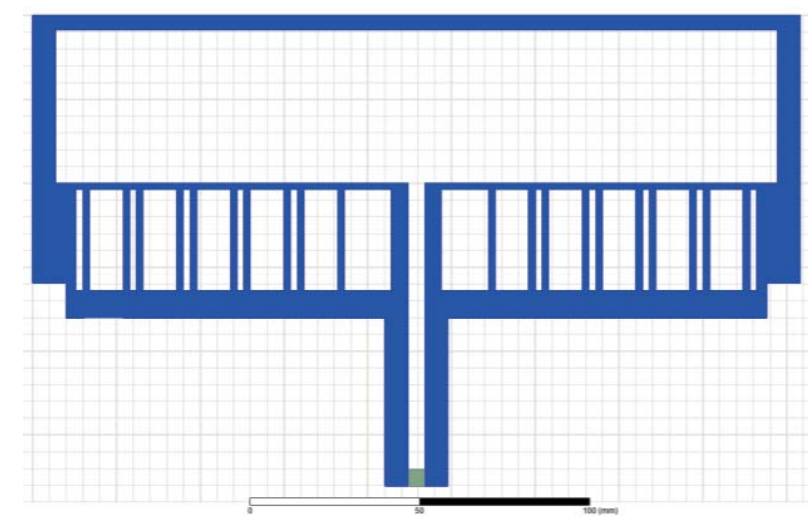

Fig. 26. Modified loop antenna with meander line electrical dipole antenna.

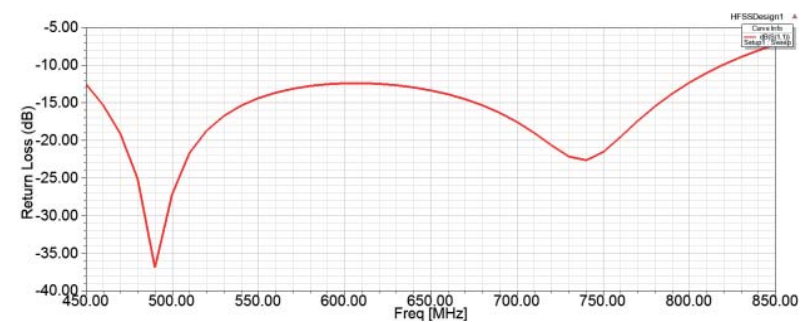

Fig. 27. Return loss for modified loop antenna with meander line electrical dipole antenna.

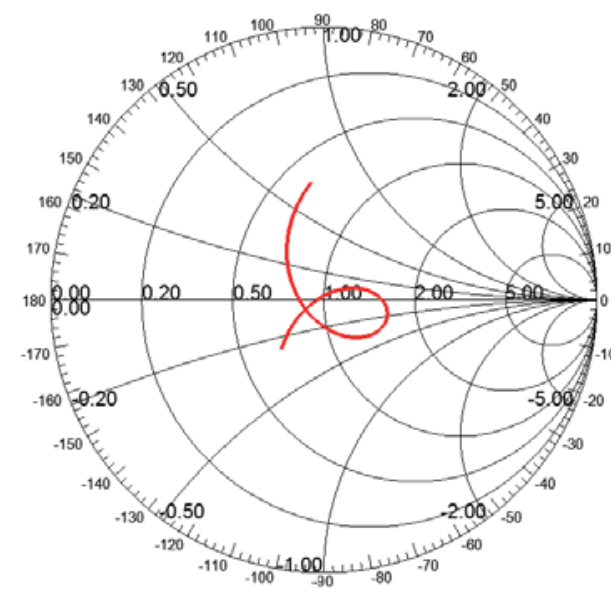

Fig. 28. Modified loop antenna with meander line electrical dipole antenna Smith chart.

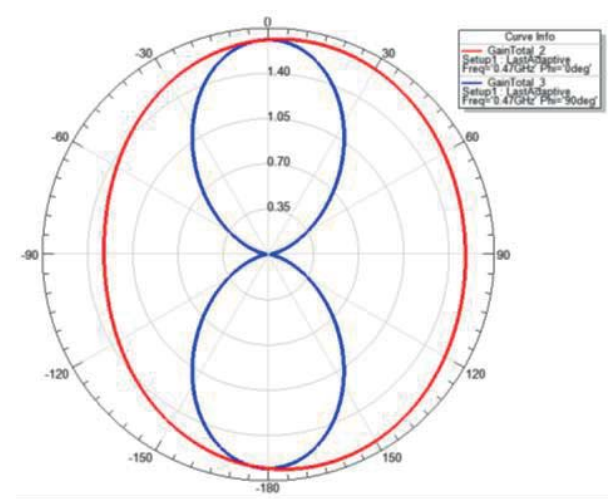

Fig. 29. Modified loop antenna with meander line electrical dipole antenna radiation pattern for $470 \mathrm{MHz}$. 


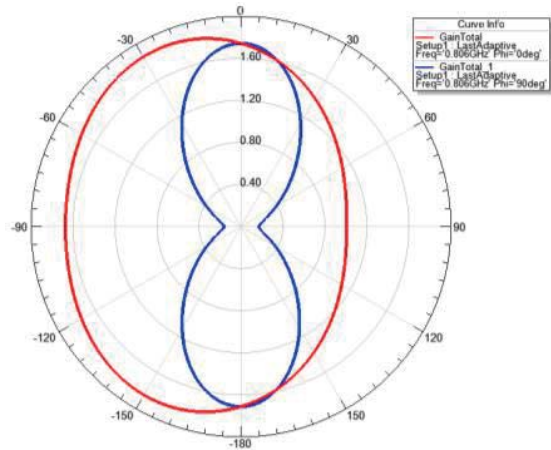

Fig. 30. Modified loop antenna with meander line electrical dipole antenna radiation pattern for $806 \mathrm{MHz}$.

As shown in Fig. 30, the radiation patterns of the loop antenna modified with meander line are very similar to those of the modified loop antenna.

\section{COMPARISON BETWEEN THE Four PRESENTED ANTENNAS}

A graph comparing the return loss for the four antennas presented in this paper is presented in Fig. 31. It can be observed that both modified loop antenna and modified loop antenna with meander line satisfy the criterion of having a return loss below $-10 \mathrm{~dB}$ for the frequency range used for HDTV. The original loop antenna and the electric dipole meander line antenna satisfy the criteria only for a short frequency range.

Therefore, for an application where the amount of material used is one of the criteria for choosing the antenna, the modified loop antenna with meander line would be the best choice, because besides having a good return loss curve, it uses less material than the modified loop antenna. For an application where the complexity of the antenna manufacturing, such as the amount of cuts that must be made in the material used, is an important characteristic, the modified loop antenna would be the best option.

Still in a criterion of material saving for manufacturing and cost reduction, the meander line antenna developed is presented as a viable option, even with technical characteristics slightly lower than the antenna presented previously.

Fig. 6, 7, 17, 18, 24, 25, 29 and 30 show the radiation patterns and hence the gain of the antenna loop original, modified loop, meander line and modified loop with meander line. It is possible to note that the maximum gain is practically the same for all antennas. However the modified loop and modified loop antenna with meander line have a reduced gain in the direction of the terminal with increased length.

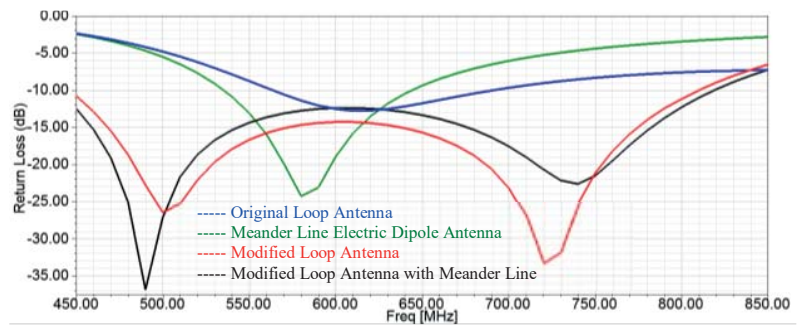

Fig. 31. Comparison between the return losses of the four antennas presented.
Thus, for an application in which the closest proximity to an omnidirectional antenna is desired, the original loop antenna and the meander line antenna are the best options.

\section{CONCLUSION}

In this paper was presented the behavior for four different antennas in the frequency range used for HDTV through numerical simulations. Depending on the manufacturing process available and the costs involved, it can be chosen one of the configurations presented, maintaining to a large extent the expected technical performance.

\section{REFERENCES}

[1] Balanis, C.A., "Wire Elements: Dipoles, Monopoles and Loops" in Modern Antenna Handbook, USA, 2008

[2] John L. Volakis; Arindam Chatterjee; Leo C. Kempel, "Overview of the Finite Element Method: OneDimensional Examples," in Finite Element Method Electromagnetics:Antennas, Microwave Circuits, and Scattering Applications , 1, Wiley-IEEE Press, 1998, pp.65-92

[3] (2017). ANSYS HFSS website. [Online]. Available: http://www.ansys.com/products/electronics/ansys-hfss

[4] Matthew N. O. Sadiku, Elements of Electromagnetics, 3rd ed., 2000

[5] Elya B. Joffe; Kai-Sang Lock, "Appendix F: Overview of S Parameters," in Grounds for Grounding:A Circuit to System Handbook, 1, Wiley-IEEE Press, 2010, pp.1045-1055

[6] de Moura, D., Cezário, I., Raizer, A.; 2015. Desenvolvimento de Sistema de Recepção com Amplificação do Nível de Sinal Digital de Alta Definição. SET EXPO PROCEEDINGS. ISNN Print: 24470481.ISSN Online: 2447-049X. v.i.doi: $10.18580 /$ setep.2015.1.13 Web-link: http://dx.doi.org/10.18580/setep.2015.1.13

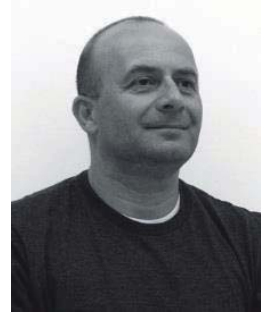

Adroaldo Raizer, was born in Lages, Santa Catarina, Brazil, on August 11, 1963. He received the titles of Electrical Engineer (1985) and master's degree in electrical engineering (1987), both from the Federal University of Santa Catarina. Completed his doctorate (1991) in Electrical Engineering by the Institut National Polytechnique de Grenoble, France. Currently Dr. Raizer is a full professor of the Department of Electrical and Electronic Engineering of the Federal University of Santa Catarina. He also works as coordinator of the Electromagnetic Compatibility and Engineering Group (GEMCO) and the Electromagnetic Compatibility and Electromagnetic Compatibility Laboratory (MagLab). His areas of interest are electromagnetic fields, electromagnetic compatibility and numerical methods.

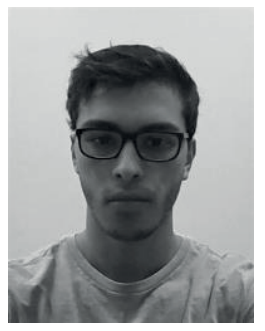

Lucas Biava Lazare, was born in Salto Veloso, Santa Catarina, Brazil, on September 19, 1995. He is currently studying electrical engineering at Federal University of Santa Catarina (UFSC) and doing an internship at Electromagnetism and Electromagnetic Compatibility Laboratory (MagLab). His areas of interest are electromagnetic fields, electromagnetic compatibility, automation and embedded systems.

Received in 2017-07-31 | Approved in 2017-11-07 